\title{
Kinetic Monte Carlo Simulations of Crystal Growth in Ferroelectric Alloys
}

\author{
Malliga Suewattana, Henry Krakauer, and Shiwei Zhang \\ Department of Physics, College of William and Mary \\ Williamsburg, VA 23187 \\ (Dated: November 20, 2018)
}

\begin{abstract}
The growth rates and chemical ordering of ferroelectric alloys are studied with kinetic Monte Carlo (KMC) simulations using an electrostatic model with long-range Coulomb interactions, as a function of temperature, chemical composition, and substrate orientation. Crystal growth is characterized by thermodynamic processes involving adsorption and evaporation, with solid-on-solid restrictions and excluding diffusion. A KMC algorithm is formulated to simulate this model efficiently in the presence of long-range interactions. Simulations were carried out on $\mathrm{Ba}\left(\mathrm{Mg}_{1 / 3} \mathrm{Nb}_{2 / 3}\right) \mathrm{O}_{3}(\mathrm{BMN})$ type materials. Compared to the simple rocksalt ordered structures, ordered BMN grows only at very low temperatures and only under finely tuned conditions. For materials with tetravalent compositions, such as $(1-x) \mathrm{Ba}\left(\mathrm{Mg}_{1 / 3} \mathrm{Nb}_{2 / 3}\right) \mathrm{O}_{3}+x \mathrm{BaZrO}_{3}(\mathrm{BMN}-\mathrm{BZ})$, the model does not incorporate tetravalent ions at low-temperature, exhibiting a phase-separated ground state instead. At higher temperatures, tetravalent ions can be incorporated, but the resulting crystals show no chemical ordering in the absence of diffusive mechanisms.
\end{abstract}

\section{INTRODUCTION}

Ferroelectric crystals are known for their important technological applications such as high-permitivity dielectrics, piezoelectric sensors, transducers, and mechanical actuators $\stackrel{1}{\underline{1}}$ Recently, single-crystal relaxor perovskites such as $\mathrm{Pb}\left(\mathrm{Zn}_{1 / 3} \mathrm{Nb}_{2 / 3}\right) \mathrm{O}_{3}-\mathrm{PbTiO}_{3}$ (PZN-PT) and $\mathrm{Pb}\left(\mathrm{Mg}_{1 / 3} \mathrm{Nb}_{2 / 3}\right) \mathrm{O}_{3}-\mathrm{PbTiO}_{3}$ (PMN-PT) were synthesized and found to exhibit ultrahigh stain and very large piezoelectric constants ${ }^{2}$ The structure of alloys like PMN-PT can be viewed as a perovskite $\mathrm{ABO}_{3}$ framework (a cubic lattice for the ideal perovskite crystal), with $\mathrm{Pb}$ ions on the A-site and a solid solution of $\left(\mathrm{Mg}^{+2}, \mathrm{Nb}^{+5}\right.$, $\mathrm{Ti}^{+4}$ ) ions on the B-sites, with average +4 B-site ionic charge. Of course this is an idealized picture, neglecting vacancies, impurities, local structural distortions, and partial chemical ordering on the B-sites.

Partial B-site chemical ordering is a common feature of the high-piezoelectric solid solutions. While random B-site ordering is observed in isoelectronic solid solutions like $\mathrm{Pb}\left(\mathrm{Zr}_{1-x} \mathrm{Ti}_{x}\right) \mathrm{O}_{3}$ (PZT), non-isoelectronic Bsite solid solutions $A\left(\mathrm{BB}^{\prime} \mathrm{B}^{\prime \prime}\right) \mathrm{O}_{3}$, with $\mathrm{B}$-site cations from group II, IV, and V, often exhibit compositionallydependent B-site chemical ordering. At $1640^{\circ} \mathrm{C}$, when the tetravalent composition $x$ is increased in (1$x) \mathrm{Ba}\left(\mathrm{Mg}_{1 / 3} \mathrm{Nb}_{2 / 3}\right) \mathrm{O}_{3}+x \mathrm{BaZrO}_{3}$ (BMN-BZ), the following sequence of B-site ordering is observed: $[111]_{1: 2}$ order for $x<5 \%$; then $[111]_{1: 1}$ order for $5 \%<x<25 \%$; and finally disorder for larger $x \underline{\underline{\underline{3}}}$ The $[111]_{1: 2}$ notation refers to x-ray observation of alternating $\beta \beta \beta^{\prime}$ [111] stacking of B-sites, where $\beta$ and $\beta^{\prime}$ denote average scattering sites. For example, in BMN-BZ with $x=0$, one can identify $\beta$ with $\mathrm{Nb}$ and $\beta^{\prime}$ with $\mathrm{Mg}$. The $[111]_{1: 1}$ notation refers to $\mathrm{x}$-ray observation of rocksaltlike alternating $\beta \beta^{\prime}$ [111] stacking of B-cations. In this case, the assignment of the $\beta$ and $\beta^{\prime}$ sites has been debated, as discussed below in connection with the spacecharge and random-site models ${ }^{3}$. Other Ba-based perovskites, e.g., (1-x) $\mathrm{Ba}\left(\mathrm{Mg}_{1 / 3} \mathrm{Ta}_{2 / 3}\right) \mathrm{O}_{3}+x \mathrm{BaZrO}_{3}$
(BMT-BZ) ${ }_{\underline{3}}^{\underline{3}}(1-x) \quad \mathrm{Ba}\left(\mathrm{Mg}_{1 / 3} \mathrm{Nb}_{2 / 3}\right) \mathrm{O}_{3}+x \quad \mathrm{BaZrO}_{3}$

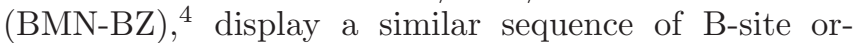
der. On the other hand, for Pb-based systems, e.g., (1x) $\mathrm{Pb}\left(\mathrm{Mg}_{1 / 3} \mathrm{Ta}_{2 / 3}\right) \mathrm{O}_{3}+x \mathrm{PbZrO}_{3}$ (PMT-PZ), [111] $]_{1: 2}$ order is not observed at $x=0$; instead, annealing between $1325^{\circ} \mathrm{C}$ and $1350^{\circ} \mathrm{C}$ results in $[111]_{1: 1}$ order all the way down to $x=0, \frac{5.6}{5}$ Other Pb-based perovskites, e.g., $\mathrm{Pb}\left(\mathrm{Mg}_{1 / 3} \mathrm{Nb}_{2 / 3}\right) \mathrm{O}_{3}(\mathrm{PMN}) \stackrel{7.8}{=}$ display similar B-site ordering.

Since their discovery, growing large single crystals has been a major research goal, but this effort has been largely unsupported by theory, because of the difficulty in modelling and simulating the non-equilibrium processes occurring in nucleation and crystal growth in such complex materials. In this paper, we use kinetic Monte Carlo ${ }^{\frac{9}{}}$ simulations of a simple effective Hamiltonian to model the growth process of these ferroelectric crystals.

Given the ionic character of these materials, it is not surprising that the inclusion of Coulomb interactions has been found to be crucial in describing their properties. A simple, purely electrostatic model introduced by Bellaiche and Vanderbilt $(\mathrm{BV})^{10}$ has had considerable success in explaining the observed equilibrium Bsite chemical ordering in many perovskite alloys. The BV model only considers Coulomb interactions between point charges $(+2,+5,+4$, etc., representing the different atomic species) that reside on the B-sites, which are constrained to lie on an ideal cubic sublattice.

This electrostatic model is the starting point of our growth simulations. Simplified models based on Ising like effective Hamiltonians $H_{\text {eff }}$ have been used to model growth in simpler systems 11.12 . These models often have only short-range interactions. To adapt the electrostatic model of BV to study crystal growth, we consider a slab-geometry with periodic boundary conditions in twodimensions only. The slab is viewed as being embedded in a liquid-phase melt, which is parametrized by a chemical potential difference with the solid bulk phase. In our simulations, a solid-on-solid (SOS) restriction is im- 
posed, which requires that adsorption only occur onto empty lattice sites directly above an occupied site, so void formation is neglected. In keeping with the simplicity of the model, diffusion in the bulk and at the surface is also neglected.

The non-equilibrium dynamics of the growth process is modeled using the kinetic Monte Carlo (KMC) method 13 . The KMC algorithm introduced by Bortz, Kalos, and Lebowitz (BKL) $)^{9}$ has been quite successful in simulating crystal growth in Ising-like models with short-range interactions between adatoms. We generalize the algorithm to efficiently handle the long-range interactions in the BV model.

In this paper we study the properties of this minimal paradigm of the growth process to determine if it can yield insights into the physics of the observed B-site chemical ordering. Section II presents our theoretical approach. The model of BV is reviewed, and our adaptation of it for the growth modeling is discussed, including the special handling of electrostatic interactions during the growth process. Our generalization and modification of the KMC algorithm are then described which allows efficient treatment of the long-range Coulomb interactions. Section III presents the results of our growth simulations for $\mathrm{A}\left(\mathrm{BB}^{\prime}\right) \mathrm{O}_{3}$ and $\mathrm{A}\left(\mathrm{BB}^{\prime} \mathrm{B}^{\prime \prime}\right) \mathrm{O}_{3}$ crystals. To help understand our growth results for the latter systems, where a (typically small) fraction of tetravalent $\mathrm{B}^{\prime \prime}$ ions are mixed in, we also carry out total energy calculations to study their stability. Finally in section IV we further discuss our results and prospects for the model. In the appendix, we include some technical details on the treatment of the long-range interactions in our simulations. A preliminary account of part of this work has already appeared 14 .

\section{THEORETICAL APPROACH}

At each stage of the simulation the crystal is modeled as a slab of finite thickness. However, it is convenient to index the allowed B-sites as in an infinite threedimensional crystal lattice

$$
\boldsymbol{l}=i \boldsymbol{a}_{1}+j \boldsymbol{a}_{2}+k \boldsymbol{a}_{3} .
$$

Two-dimensional (2-D) periodic boundary conditions (PBC) are employed along the $\boldsymbol{a}_{1}$ and $\boldsymbol{a}_{2}$ directions, which define the $x-y$ Cartesian plane, using a $L_{1} \boldsymbol{a}_{1} \times L_{2} \boldsymbol{a}_{2}=\boldsymbol{A}_{1} \times \boldsymbol{A}_{2} 2$-D supercell. Growth proceeds along the $z$-direction. The simulation is initialized as a slab of uniform thickness $H_{0} \boldsymbol{a}_{3}$, with a predefined B-atom configuration. A given simulation is terminated when either the maximum slab thickness or the maximum number of Monte Carlo (MC) time steps is reached. We use the notation $L \times L \times H_{\max }$ to label a particular simulation, where $H_{\max }$ is the number of layers for maximum slab thickness (the initial substrate included).

The SOS restriction that we impose does not allow the formation of voids. The crystal configuration, $\mathcal{C}$, is specified at each stage of the simulation by the set of occupied sites $\boldsymbol{l}=(i, j, k)$ and their charges $q_{\boldsymbol{l}}$. The BV electrostatic model cannot be directly used in this slab geometry, due to ill-defined electrical boundary conditions in the $z$ direction and the lack of exact charge neutrality during the growth simulation. Section A describes how we handle these issues. Similarly, a direct application of the KMC algorithm is inefficient due to the long-range Coulomb interaction. Section B describes the KMC method and our modifications to make it applicable to the model.

\section{A. The Electrostatic Model}

The BV model is derived by considering the total electrostatic energy for an $\mathrm{A}\left(\mathrm{BB}^{\prime} \mathrm{B}^{\prime \prime}\right) \mathrm{O}_{3}$ compound:

$$
E(\mathcal{C})=\sum_{\left(\boldsymbol{l} \tau, \boldsymbol{l}^{\prime} \tau^{\prime}\right)} \frac{Q_{\boldsymbol{l} \tau} Q_{\boldsymbol{l}^{\prime} \tau^{\prime}}}{\epsilon\left|\boldsymbol{R}_{\boldsymbol{l} \tau}-\boldsymbol{R}_{\boldsymbol{l}^{\prime} \tau^{\prime}}\right|},
$$

where $\boldsymbol{R}_{\boldsymbol{l} \tau}$ is the position of the ion on site $\tau\left(=\mathrm{A}, \mathrm{B}, \mathrm{O}_{1}\right.$, $\mathrm{O}_{2}, \mathrm{O}_{3}$ ) in cell $\boldsymbol{l}$, and $\epsilon$ is the dielectric constant. For a given Bravais lattice, $\epsilon$ sets the energy scale. We consider the perovskite structure with group II A-site atoms (e.g. $\mathrm{Ba}, \mathrm{Pb}$ ), so the charges on the $\mathrm{A}$ and $\mathrm{O}$ sites have fixed values of $+2 e$ and $-2 e$, respectively. Since the average Bsite charge is $+4 e$, it is convenient to express the charges on the B-sites, $Q_{l, B}$, as

$$
Q_{l, B}=4 e+q_{l} .
$$

Up to a constant, the configurationally averaged electrostatic energy depends only on the B-site charges, since the configurational average of $q_{l}$ is zero:

$$
E_{B}(\mathcal{C})=\frac{1}{\epsilon a} \sum_{\left(\boldsymbol{l}, \boldsymbol{l}^{\prime}\right)} \frac{q_{\boldsymbol{l}} q_{\boldsymbol{l}^{\prime}}}{\left|\boldsymbol{l}-\boldsymbol{l}^{\prime}\right|},
$$

where we have for simplicity restricted ourselves in Eq. (4) to a cubic Bravais lattice with lattice parameter $a$, and $\boldsymbol{R}_{l B}=\boldsymbol{l} a$. In this model each cell $\boldsymbol{l}$ is therefore reduced to a single lattice site with charge $q_{l}$, and the energy of the compound is given by the inter-site Coulomb interaction.

The long-range Coulomb interaction must be treated with care in a bulk simulation to ensure proper convergence. For 2-D and 3-D simulations with periodic boundary conditions, the method of Ewald is often used, in which periodic images of the charges and neutralizing background charges are introduced ${ }^{15.16 .17 .18 .19}$ so that the bare Coulomb form $1 /\left|\boldsymbol{l}-\boldsymbol{l}^{\prime}\right|$ is replaced with a reduced form $v\left(\boldsymbol{l}-\boldsymbol{l}^{\prime}\right)$. For our growth simulations, we are dealing with a slab geometry with PBC only in two dimensions $(x-y)$. Some modifications are required before the Ewald method can be applied.

In the simulations, we will need to calculate the energy change from Eq. (4) due to the evaporation of a charged ion $q_{l^{\prime}}$ at the surface of the crystal (see Eq. (25) below). 
The distribution of point charges that $q_{l^{\prime}}$ "sees" can be described by the charge density

$$
\rho(\boldsymbol{r})=\sum_{\boldsymbol{l}} \sum_{\boldsymbol{R}} q_{\boldsymbol{l}} \delta(\boldsymbol{r}-\boldsymbol{l}-\boldsymbol{R})
$$

where $\boldsymbol{l}$ runs through the position vectors of the atoms within the simulation cell, and $\boldsymbol{R}$ is a 2-D Bravais supercell lattice vector: $\boldsymbol{R}=n_{1} \boldsymbol{A}_{1}+n_{2} \boldsymbol{A}_{2}$. Directly summing the Coulomb potentials of the individual point charges, $V(\boldsymbol{r}-\boldsymbol{l}-\boldsymbol{R})=q_{\boldsymbol{l}} /|\boldsymbol{r}-\boldsymbol{l}-\boldsymbol{R}|$, leads to an ill-defined and conditionally convergent result, as is well known. However, for three-dimensional periodic boundary conditions, a unique solution of Poisson's equation exists (for an electrically neutral system), and it is conveniently calculated using Ewald's method. Subject to some additional, physically motivated conditions, a unique solution can also be found for finite thickness slabs that are infinite in extent along two spatial directions.

Solutions of Poisson's equation, $\nabla^{2} V(\boldsymbol{r})=-4 \pi \rho(\boldsymbol{r})$, in our simulations are subject to two-dimensional (2-D) PBC $V(\boldsymbol{r}+\boldsymbol{R})=V(\boldsymbol{r})$, as is the charge density $\rho(\boldsymbol{r})$. The 2-D PBC imply that $V(\boldsymbol{r})$ and $\rho(\boldsymbol{r})$ can be expanded as:

$$
\begin{aligned}
& \rho(\boldsymbol{r})=\sum_{\boldsymbol{G}} \rho_{\boldsymbol{G}}(z) e^{i \boldsymbol{G} \cdot \boldsymbol{r}_{p}} \\
& V(\boldsymbol{r})=\sum_{\boldsymbol{G}} V_{\boldsymbol{G}}(z) e^{i \boldsymbol{G} \cdot \boldsymbol{r}_{p}},
\end{aligned}
$$

where $\boldsymbol{G}$ is a 2-D supercell reciprocal lattice vector and $\boldsymbol{r}_{p}$ is the $x-y$ component of $\boldsymbol{r}, \boldsymbol{r}_{p}=\boldsymbol{r}-(\boldsymbol{r} \cdot \hat{\boldsymbol{z}}) \hat{\boldsymbol{z}}=i \boldsymbol{a}_{1}+j \boldsymbol{a}_{2}$. Substitution of Eqs. (6) into Poisson's equation yields the ordinary differential equation

$$
\frac{d^{2} V_{\boldsymbol{G}}(z)}{d z^{2}}-G^{2} V_{\boldsymbol{G}}(z)=-4 \pi \rho_{\boldsymbol{G}}(z),
$$

whose solution can be expressed as

$$
V_{\boldsymbol{G}}(z)=-4 \pi \int_{-\infty}^{\infty} \mathcal{G}\left(z-z^{\prime}\right) \rho_{\boldsymbol{G}}\left(z^{\prime}\right) d z^{\prime}
$$

where $\mathcal{G}\left(z-z^{\prime}\right)$ is the Green's function corresponding to Eq. (17).

If there are any ill-defined contributions to the Coulomb potential, they must arise from the $\boldsymbol{G}=\mathbf{0}$ solution in Eq. (77) or (8). This is because only the $\boldsymbol{G}=\mathbf{0}$ term of $\rho(\boldsymbol{r})$ in Eqs. (6) contributes to the net slab charge. Even if the slab is electrically neutral, there may still be a net dipole moment $D$, which would lead to different asymptotic values of Coulomb potential at $z= \pm \infty$. Again, $D$ also depends only on the $\boldsymbol{G}=\mathbf{0}$ term of $\rho(\boldsymbol{r})$, where

$$
D \equiv \int_{-\infty}^{\infty} z \bar{\rho}(z) d z
$$

with

$$
\bar{\rho}(z)=\frac{1}{A} \int_{A} \rho(\boldsymbol{r}) d x d y=\rho_{\boldsymbol{G}=\mathbf{0}}(z),
$$

where $A$ is the area of the 2-D supercell.

We therefore first consider the well-defined $\boldsymbol{G} \neq \mathbf{0}$ solutions of Eq. (7). Physically meaningful results require that the solutions satisfy $\lim _{|z| \rightarrow \infty} V_{G}(z)=0$, which leads to the following unique definition of the $\boldsymbol{G} \neq \mathbf{0}$ Green's function:

$$
\mathcal{G}\left(z-z^{\prime}\right) \equiv-\frac{\left[\vartheta\left(z-z^{\prime}\right) e^{-G\left(z-z^{\prime}\right)}+\vartheta\left(z^{\prime}-z\right) e^{G\left(z-z^{\prime}\right)}\right]}{2 G},
$$

where $G=|\boldsymbol{G}|$. For any reasonably localized charge distribution $\rho_{\boldsymbol{G}}(z)$, Eqs. (8) and (11) result in well-behaved, exponentially decaying solutions of $V_{\boldsymbol{G}}(z)$ as $|z| \rightarrow \infty$.

For $\boldsymbol{G}=\mathbf{0}$, Eq. (7) becomes

$$
\frac{d^{2} V_{\mathbf{0}}(z)}{d z^{2}}=-4 \pi \rho_{\mathbf{0}}(z)
$$

As adatoms are adsorbed or atoms evaporate in the course of the growth simulations, the net charge will fluctuate so that the total charge in the simulation supercell will not be precisely zero at each stage of the simulation. Similarly a net dipole $D$ may form. However, in a real growth process there are always compensating charges that will cancel any ill-defined long-range effects due to the lack of charge neutrality or the presence of a dipole moment. In our calculations, we simulate this by a construction that ensures that $\rho_{0}(z)$ in Eq. (12) always represents a neutral charge distribution with $D=0$. This leads to well-defined boundary conditions $\lim _{|z| \rightarrow \infty} V_{\mathbf{0}}(z)=0$.

As in the 3-D Ewald method, a diffuse localized charge density $g(\boldsymbol{r})$ is added and subtracted to each point charge to facilitate the decomposition of the potential into absolutely convergent direct- and reciprocal lattice sums:

$$
\begin{aligned}
\rho(\boldsymbol{r}) & =\sum_{\boldsymbol{l}} \sum_{\boldsymbol{R}} q_{\boldsymbol{l}}[\delta(\boldsymbol{r}-\boldsymbol{l}-\boldsymbol{R})-g(\boldsymbol{r}-\boldsymbol{l}-\boldsymbol{R})]+\sum_{\boldsymbol{l}} \sum_{\boldsymbol{R}} q_{l} g(\boldsymbol{r}-\boldsymbol{l}-\boldsymbol{R}) \\
& \equiv \rho_{1}(\boldsymbol{r})+\rho_{2}(\boldsymbol{r}) .
\end{aligned}
$$

The diffuse charge density $g(\boldsymbol{r})$ is chosen to be a normal-

ized spherically symmetric Gaussian, as in the 3-D Ewald 
method:

$$
g(\boldsymbol{r}) \equiv\left(\frac{\alpha}{\pi}\right)^{3 / 2} e^{-\alpha r^{2}}
$$

where the value of the Ewald convergence parameter $\alpha$ is arbitrary, but is usually chosen to optimize the convergence of both the direct- and reciprocal-lattice sums. The integrated charge of $\rho_{1}(\boldsymbol{r})$ is zero by construction, as is its dipole moment $D$, so its contribution $V_{1}(\boldsymbol{r})$ to the Coulomb potential can be obtained by a rapidly convergent direct-lattice sum, given in the Appendix.

The procedure for calculating the Coulomb potential $V_{2}(\boldsymbol{r})$ due to $\rho_{2}(\boldsymbol{r})$ requires special handling. $V_{2}\left(\boldsymbol{l}^{\prime}\right)$, the potential at the position of $q_{l^{\prime}}$ in the simulation cell, is due to: $i$ ) the $\boldsymbol{l} \neq \boldsymbol{l}^{\prime}$ Gaussian charge densities and their periodic images $q_{\boldsymbol{l}} g(\boldsymbol{r}-\boldsymbol{l}-\boldsymbol{R})$, and $\left.i i\right)$ the periodic images $q_{\boldsymbol{l}^{\prime}} g\left(\boldsymbol{r}-\boldsymbol{l}^{\prime}-\boldsymbol{R}\right)$. [As in the 3D Ewald method, a spurious interaction of the point charge $q_{l^{\prime}}$ with its own Gaussian density $q_{\boldsymbol{l}^{\prime}} g\left(\boldsymbol{r}-\boldsymbol{l}^{\prime}\right)$ is explicitly removed later.] Alternatively, the contribution (ii) above can be replaced by iia) the Gaussian densities $-q_{l} g\left(\boldsymbol{r}-\boldsymbol{l}^{\prime}-\boldsymbol{R}\right)$ located at the positions of the periodic images of $\boldsymbol{l}^{\prime}$. In a bulk crystal simulation with $3-\mathrm{D}$ PBC and a neutral simulation cell, these two formulations are equivalent, since the integrated total charge vanishes:

$$
\sum_{l \neq l^{\prime}}-q_{\boldsymbol{l}}=q_{\boldsymbol{l}^{\prime}}
$$

In the 2-D slab geometry of our growth simulations, this will not be the case in general. Overall charge neutrality is still satisfied in a statistical sense, however. Our procedure for calculating $V_{2}(\boldsymbol{r})$ consists of two approximations. The first approximation is to use formulation (iia) above. This means that the contribution of each $q_{l} g(\boldsymbol{r})$ sublattice to $V_{2}\left(\boldsymbol{l}^{\prime}\right)$ is to be calculated as the potential due to the charge density:

$$
\rho_{2}^{\left(\boldsymbol{l}, \boldsymbol{l}^{\prime}\right)}(\boldsymbol{r})=q_{\boldsymbol{l}} \sum_{R}\left[g(\boldsymbol{r}-\boldsymbol{l}-\boldsymbol{R})-g\left(\boldsymbol{r}-\boldsymbol{l}^{\prime}-\boldsymbol{R}\right)\right] .
$$

Since the integrated charge of $\rho_{2}^{\left(l, l^{\prime}\right)}(\boldsymbol{r})$ is zero, the use of this approximation effectively imposes overall charge neutrality at each stage of the growth simulation.

The boundary conditions are still ill-defined however, since the sum of sublattice potentials due to the $\rho_{2}^{\left(l, l^{\prime}\right)}(\boldsymbol{r})$ may still have a dipole moment $D$. We therefore introduce a second approximation: the Gaussian image densities $-q_{l} g\left(\boldsymbol{r}-\boldsymbol{l}^{\prime}-\boldsymbol{R}\right)$ are made coplanar with the $q_{l} g(\boldsymbol{r}-\boldsymbol{l}-\boldsymbol{R})$ sublattice. In other words, the Gaussian densities $-q_{l} g(\boldsymbol{r})$ are placed at positions that are the projections of the $q_{l}$, image positions onto the plane defined by the $q_{l}$ sublattice. In place of Eq. (16), the contribution of each $q_{l} g(\boldsymbol{r})$ sublattice is thus calculated as the potential due to the charge density:

$$
\tilde{\rho}_{2}^{\left(l, \boldsymbol{l}^{\prime}\right)}(\boldsymbol{r})=q_{\boldsymbol{l}} \sum_{R}\left[g(\boldsymbol{r}-\boldsymbol{l}-\boldsymbol{R})-g\left(\boldsymbol{r}-\tilde{\boldsymbol{l}}^{\prime}-\boldsymbol{R}\right)\right],
$$

where $\tilde{\boldsymbol{l}}^{\prime}$ denotes the projection of the position $\boldsymbol{l}^{\prime}$ onto the plane defined by the $q_{l}$ sublattice. The charge density $\tilde{\rho}_{2}^{\left(l, l^{\prime}\right)}(\boldsymbol{r})$ has a rapidly convergent expansion in terms of 2-D planewaves given by Eq. (6). Moreover, the $\boldsymbol{G}=0$ contribution of $\tilde{\rho}_{2}^{\left(l, l^{\prime}\right)}(\boldsymbol{r})$ vanishes, so the Coulomb potential $V_{2}(\boldsymbol{r})$ is readily found using Eqs. (8) and (11). These two approximations ensure overall average-charge neutrality and vanishing dipole moment $D=0$, resulting in a well-defined Coulomb potential at each stage of the growth simulation. Complete formulas for the potential $v\left(\boldsymbol{l}^{\prime}-\boldsymbol{l}\right)$ are given in the Appendix.

\section{B. Kinetic Monte Carlo method for long-range interactions}

The kinetic Monte Carlo (KMC) method is one of several simulation techniques commonly employed to model the relaxation processes of systems away from equilibrium (e.g. growth processes). It has been applied successfully to crystal growth and surface/interface

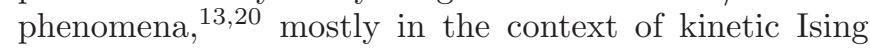
models. Due to the long-range interactions between ions in our electrostatic model, the usual implementation of $\mathrm{KMC}$ for Ising-like models is inefficient, with the acceptance rates of events becoming very low. We developed a modified sampling algorithm to make the simulation practical for this model. Here we briefly outline the basic theoretical background for the KMC method, and then describe our modifications and give the relevant implementation details.

In the KMC simulation, the dynamics of the system is described as stochastic processes such as adsorption, evaporation, and surface migration. We consider only the first two in our simulation. As mentioned, the adatoms represent the B-site ions in the single crystal perovskite alloy. They are characterized entirely by their charges and they interact with each other by the interaction described above.

In the grand canonical ensemble, the Hamiltonian that will be used in the growth simulations can then be expressed in term of Eq. (4) as

$$
\mathcal{H}(\mathcal{C})=E_{B}(\mathcal{C})+\Delta \mu N,
$$

where $N$ is the total number of adsorbed adatoms. The electrostatic energy term in the Hamiltonian is responsible for evaporation, while the second term, which depends on the chemical potential difference between the solid and the gas phases, controls the rate in which adatoms stick on the surface. The growth simulation is then characterized by competing adsorption and desorption events. The SOS restriction imposed in the simulation prevents formation of vacancies and allows us to write $H$ as

$$
\mathcal{H}(\mathcal{C})=E_{B}(\mathcal{C})+\Delta \mu \sum_{i, j} h_{i j}
$$


where $h_{i j}$ is the number of layers in the present crystal configuration at the horizontal position $i \boldsymbol{a}_{1}+j \boldsymbol{a}_{2}$.

In $\mathrm{KMC}$ the time evolution of the system is simulated through a Markov chain of configurations. Let us define $P(C, t)$ as a time-dependent distribution of configurations. The transition rate from $C$ to $C^{\prime}$, a crystal configuration related to $C$ by a single time step, is denoted by $w\left(C \rightarrow C^{\prime}\right)$. We then have the usual master equation 20 :

$$
\begin{aligned}
\frac{\partial P(C, t)}{\partial t}= & -\sum_{C^{\prime}} w\left(C \rightarrow C^{\prime}\right) P(C, t) \\
& +\sum_{C^{\prime}} w\left(C^{\prime} \rightarrow C\right) P\left(C^{\prime}, t\right),
\end{aligned}
$$

where the first term on the right describes the loss because of transitions away from $C$, while the second term describes the gain because of transitions into $C$. In the equilibrium limit (as $t \rightarrow \infty$ ), the Boltzmann distribution

$$
P_{\text {eq }}=Z^{-1} \exp \left[\frac{-\mathcal{H}(\mathcal{C})}{k_{B} T}\right]
$$

is reached, where $k_{B}$ is the Boltzmann constant. We require that detailed balance be satisfied:

$$
\frac{w\left(C \rightarrow C^{\prime}\right)}{w\left(C^{\prime} \rightarrow C\right)}=\frac{P_{e q}\left(C^{\prime}\right)}{P_{e q}(C)}=\exp \left[-\frac{\mathcal{H}\left(C^{\prime}\right)-\mathcal{H}(C)}{k_{B} T}\right]
$$

The KMC technique can be viewed as a method of solving Eq. (20) stochastically. We adopt the following choice of transition rates $w\left(C \rightarrow C^{\prime}\right)$

$$
\begin{aligned}
& w_{\mathrm{a}}=\exp \left(\Delta \mu / k_{B} T\right) \\
& w_{\mathrm{e}}=\exp \left(-\Delta E_{B}(\mathcal{C}) / k_{B} T\right),
\end{aligned}
$$

where $w_{a}$ and $w_{e}$ are the rates for adsorption and evaporation, respectively, of an adatom. It can be verified straightforwardly that this choice indeed satisfies Eq. (22). The rate $w_{e}$ for an adatom of charge $q_{\boldsymbol{\tau}^{\prime}}$ to evaporate from the surface depends on the change in total potential energy in the crystal

$$
\begin{aligned}
\Delta E_{B}(C) & =E_{B}\left(C^{\prime}\right)-E_{B}(C) \\
& =\frac{q_{\boldsymbol{l}^{\prime}}}{\epsilon a} \sum_{\boldsymbol{l}} q_{\boldsymbol{l}} v\left(\boldsymbol{l}^{\prime}-\boldsymbol{l}\right) .
\end{aligned}
$$

For kinetic Ising models, the algorithm of BKL ${ }^{9}$ allows an efficient stochastic realization of the kinetic process under the choice in Eq.'s (23) and (24). In this algorithm, a site $(i, j)$ is selected randomly in each step at the surface of the grown crystal. An event is then selected by Monte Carlo sampling ${ }^{21}$ from the list of three possible events, \{adsorption, evaporation, nothing $\}$. The interaction in Ising type models is limited to near-neighbors, and the energy difference $\Delta E_{B}(C)$ is completely determined by the local environment at site $(i, j)$. The global maximum of $w_{e}$, i.e., the minimum possible energy change,
$\Delta E^{\text {min }}=\min \left[\Delta E_{B}(C)\right]$, can be obtained straightforwardly by considering all possible local configurations. This gives a corresponding global maximum of the evaporation rates: $w_{e}^{\max }=\exp \left(-\Delta E^{\min } / k_{B} T\right)$, which defines a normalization factor:

$$
W \equiv w_{a}+w_{e}^{\max } .
$$

The relative probabilities for the three events are therefore

$$
\left\{P_{a} \equiv \frac{w_{a}}{W}, P_{e} \equiv \frac{w_{e}}{W}, P_{n} \equiv 1-P_{a}-P_{e}\right\} .
$$

With the electrostatic model, however, the energy change in Eq. (24) depends on the entire configuration $C$. It is therefore difficult to determine the global minimum, $\Delta E^{\mathrm{min}}$. Indeed, even if $\Delta E^{\mathrm{min}}$ could be identified, the energy change $\Delta E_{B}(C)$, which can vary greatly with $C$ and the simulation cell size, would be much greater than $\Delta E^{\text {min }}$ for most configurations. This would cause the evaporation and adsorption probabilities $P_{a}$ and $P_{e}$ to be small, with $P_{n}$ approaching unity. As a result the acceptance rate of events becomes small, and the algorithm becomes ineffective.

To overcome this difficulty, we modify the standard algorithm so that all $N=L_{1} \times L_{2}$ surface sites are considered simultaneously, instead of sweeping through the surface sites. An event list is created which includes every possible event for every possible surface site. This increases the algorithm complexity, because of the need to store and update an array of surface potentials, calculate the event list, and sample an event from this list. The advantage is that an event is guaranteed to take place in each step of the algorithm and that the need for determining $\Delta E^{\text {min }}$ is eliminated. Evaporation/adsorption rates for all possible sites are normalized. The sum of the probabilities for an adsorption or evaporation to occur at a surface site is unity. Specifically, the modified algorithm consists of the following steps:

(i) Generate a list, $\mathcal{E}$, of all possible events per time step. There are $2 N$ possible events: an evaporation or an adsorption could happen on each of the $N=L_{1} \times L_{2}$ surface sites.

(ii) Calculate the rates $(w)$ of adsorption and evaporation for each site on the surface. Denote the total rates by $W: W=\sum_{i}^{2 N} w_{i}$.

(iii) Normalize these $2 N$ rates by $W$, giving probabilities, $P_{i}$, for adsorption and evaporation on sites $1,2, \cdots, 2 N$.

(iv) Generate a random number $r \in[0,1)$ and choose the first event $\mathcal{E}_{i}$ such that $\sum_{k=1}^{i} P_{k} \geq r$. An event will always be chosen.

(v) Generate the new configuration $C^{\prime}$ based on the chosen event $\mathcal{E}_{i}$. 
(vi) Assign a "real time" increment $\Delta t_{\text {real }}=-\ln \left(r^{\prime}\right) / W$ to this MC step, where $r^{\prime}$ is another random number on $[0,1)$.

The last step is a result of our considering the global event list and forcing an event to occur in every step. The issue of real "time" in a KMC simulation is a subtle one. Often the Monte Carlo time, $t_{\mathrm{MC}}$, is used as some measure of the real time. In the standard algorithm, the global normalization factor $W$ (defined by $w_{e}^{\max }$ ) controls the overall rate of events and sets a "time scale." In our approach, $W$ is time-dependent, and an event is forced to happen in each step regardless of the total rate $W$ for the configuration at hand. When $W$ is low, an evaporation or adsorption is less likely to happen but one is selected anyway. Conversely, when $W$ is high, an evaporation or adsorption is more likely to happen but still only one is selected. This introduces a bias which should vanish in the limit of large system size but which should be corrected for at finite $L$. Based on the rate equation, we assume an exponential relation between time and $W$. A step in which $W$ is high corresponds to a short time, and vice versa. Step (vi) is a way to account for this time scale stochastically, by rescaling $\Delta t_{\mathrm{MC}}$ with a $\mathrm{MC}$ sampling from an exponential distribution which is determined by the normalization factor $W$ in each step.

\section{RESULTS}

We now present the results from our simulations for $\mathrm{A}\left(\mathrm{BB}^{\prime}\right) \mathrm{O}_{3}$ and $\mathrm{A}\left(\mathrm{BB}^{\prime} \mathrm{B}^{\prime \prime}\right) \mathrm{O}_{3}$ crystals. Growth simulations are presented in Section A. Growth rates are studied, and charge-charge correlation functions are calculated to measure the degree of growth order. The effects of varying the crystallographic orientation of the slabs were explored, with the slabs labelled according to the slab perpendicular $(z)$ direction. In $\mathrm{A}\left(\mathrm{BB}^{\prime} \mathrm{B}^{\prime \prime}\right) \mathrm{O}_{3}$ systems, a fraction of tetravalent $\mathrm{B}^{\prime \prime}$ ions are mixed in. In our growth simulations, these tetravalent ions do not appear to mix at low temperatures, choosing instead to phase-separate from the pure crystal. To further study this, Section B presents results of static total energy and free-energy calculations for fixed slab configurations.

\section{A. Crystal Growth}

The growth process is a function of temperature $T$, chemical potential difference $\Delta \mu$, and the Coulomb interaction. These parameters are fixed throughout a given simulation. As discussed in Section ЩA and in the Appendix, we tabulate $v\left(\boldsymbol{l}^{\prime}-\boldsymbol{l}\right)$, and we will use reduced units in our simulations below. The energies $(\Delta \mu$ and $\left.E_{B}(C)\right)$ are scaled by $\xi \equiv 1 / \epsilon a$. There is only one free parameter between $\xi$ and the temperature $k_{B} T$, which sets the energy scale of the problem. Below we will give the temperature $k_{B} T$ in reduced units. For example, for $a \sim 8$ a.u. and $\epsilon \sim 10$ (typical values of BMN solid solutions) in Eq. (4), $1350 \mathrm{C}$ corresponds to $k_{B} T=0.41$ in the simulation.

As an overview, Figs. 1 1 and 2 present a comparison of simulations of the simple $\mathrm{III}_{1 / 2} \mathrm{~V}_{1 / 2}$ rocksalt alloy and a $\mathrm{II}_{1 / 3} \mathrm{~V}_{2 / 3}$ heterovalent alloy such as BMN. (All substrates in our simulations have neutral surface layers.) We measure the growth rate of the crystal based on the KMC dynamics. If $N_{G}$ adatoms are gained in $m$ MC steps (each defined as one attempt at the procedure outlined in Section [1]), the growth rate is defined as

$$
\Gamma=\frac{N_{G}}{w_{a} \sum_{i=1}^{m} \Delta t_{\text {real }}(i)} .
$$

Note that as defined the growth rate $\Gamma$ is renormalized by the absorption rate. The growth rate is plotted as a function of the chemical potential for a range of temperatures. The rocksalt structure has layers of positive and negative charges alternating along the [111] direction. It typifies the crystal ordering of a wide variety of materials, including some of the perovskite alloys. Heterovalent binaries, described by $\mathrm{II}_{1 / 2} \mathrm{VI}_{1 / 2}\left(q_{B}= \pm 2\right)$ or $\mathrm{III}_{1 / 2} \mathrm{~V}_{1 / 2}$ $\left(q_{B}= \pm 1\right)$, exhibit rocksalt B-site chemical order. By contrast, in the $\mathrm{II}_{1 / 3} \mathrm{~V}_{2 / 3}$ heterovalent binary BMN the equilibrium state shows $[111]_{1: 2}$ ordering of two layers of metal group $\mathrm{V}\left(q_{B}=+1\right)$ alternating with one layer of the group $\operatorname{II}\left(q_{B}=-2\right)$ atom. Both the rocksalt and BMN simulations were initialized with a 20-layer thick slab, with perfect $[111]_{1: 1}$ and $[111]_{1: 2}$ ordering, respectively. The rocksalt simulation used a 2 -D $12 \times 12$ supercell, while the BMN simulations were done mostly with $6 \times 6$ supercells, although some simulations with $12 \times 12$ and $15 \times 15$ were carried out to verify that the finite-size effects were small. The rocksalt structure simulations ran for $1,000 L^{2} \mathrm{MC}$ steps, up to a maximum thickness of 100 layers. For BMN, 10, $000 L^{2} \mathrm{MC}$ steps were used, because for a given temperature and $\Delta \mu$ growth was significantly slower. In Fig. [3 we show visualizations of the grown BMN crystals to illustrate the simulation environment and the 1:2 order at low temperatures with slow growth.

The two sets of curves in Fig 1 and 2 are qualitatively similar. What is not evident from the figures, however, is the degree of order in each simulation. For a given temperature, as $\Delta \mu$ increases, the adsorbtion rate in Eq. (23) increases, and adatoms are more likely to stick. For fixed $\Delta \mu$, as $k_{B} T$ decreases, the adsorption rate will increase, but more importantly, the "selectiveness" of evaporation will increase. A lower $k_{B} T$ will, in effect, increase the energy differences between competing configurations. The direct result, as growth is concerned, will be that adatoms will increasingly prefer to have more instead of less neighbors with correct charge ordering (layer-by-layer growth vs. rough growth), and adatoms with the same charge will seem more repulsive. For very high $\Delta \mu$, adatoms will stick anywhere, no matter what the location or ionic adversity is, and the growth rate will be high. Alternatively, if the temperature becomes too high, the crystal will melt, preferring the liquid phase, and result in neg- 


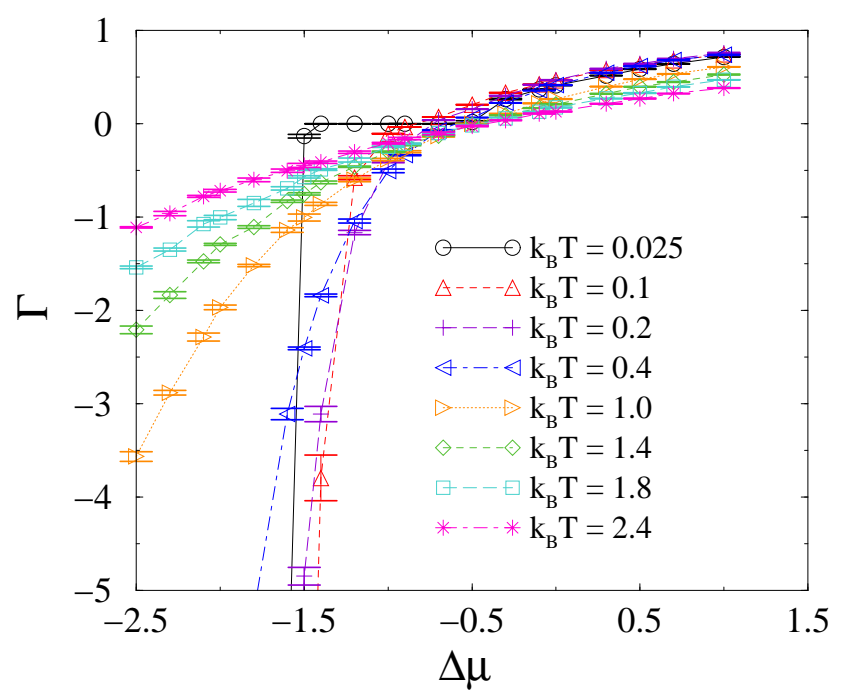

FIG. 1: Rocksalt growth rate vs. chemical potential for a [001] slab.

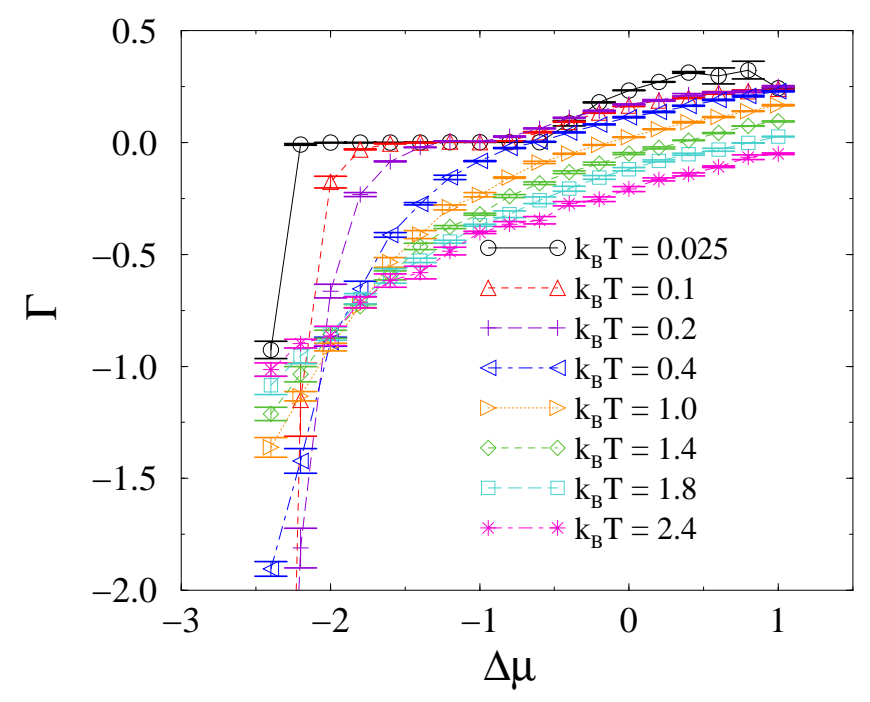

FIG. 2: BMN growth rate vs. chemical potential for a [-1-11] slab.

ative growth.

To examine the degree of ordering, we computed the charge-charge correlation function. The Fourier transform of this correlation function, which we will denote by $\eta(\boldsymbol{k})$, gives the structure factor:

$$
\eta(\boldsymbol{k})=\alpha \sum_{l l^{\prime}} q_{l} q_{\boldsymbol{l}+l^{\prime}} \exp \left(-i \boldsymbol{k} \cdot \boldsymbol{l}^{\prime}\right)
$$

where $\alpha$ is the normalization factor, and $\boldsymbol{k}$ is the wave vector in the Brillouin zone of the unit cell. The magnitude of $\eta(\boldsymbol{k})$ characterizes the B-site order, e.g., a large value of $\eta$ at $\boldsymbol{k}=\frac{2 \pi}{a}\left(\frac{1}{2}, \frac{1}{2}, \frac{1}{2}\right)$ indicates a strong [111] $]_{1: 1}$ order while one at $\boldsymbol{k}=\frac{2 \pi}{a}\left(\frac{1}{3}, \frac{1}{3}, \frac{1}{3}\right)$ indicates a strong $[111]_{1: 2}$ order.

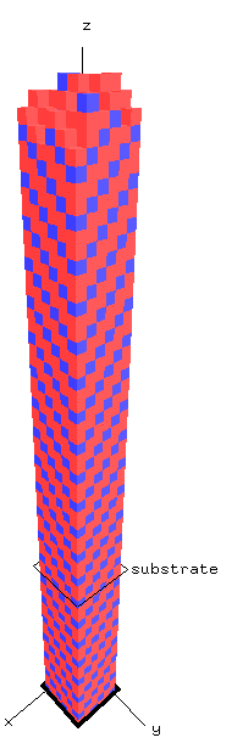

(a)

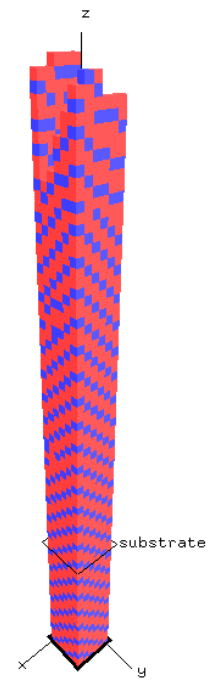

(b)
FIG. 3: Visualizations of grown BMN crystals. Shown are $6 \times$ 6 supercells with: (a) growth direction along [001], $k_{B} T=0.1$ and $\Delta \mu=-1.0$; (b) growth direction along [111], $k_{B} T=0.1$ and $\Delta \mu=-1.1$.

The growth rate and the charge-charge structure factor in Eq. 29) are plotted in Figs. 47 In each figure, the displayed range of $\Delta \mu$ was chosen to coincide with the range where the order parameter decreases from nearly unity (perfect order) to essentially zero (disorder). As $\Delta \mu$ increases the adsorption rate increases, but the growth is disordered and there is greater surface roughness. Indeed there is only a limited range where ordered growth occurs. The grown crystal structures are consistent with the observed ground state configuration of rocksalt (Fig. 4) and BMN (Fig.'s [5 7). The most striking difference between the growth behaviors of rocksalt and BMN is the enormous reduction of the growth rate of BMN compared to that of the rocksalt structure. Moreover, for rocksalt the growth rate increases linearly as a function of $\Delta \mu$ in the region where the order parameter $\eta$ is rapidly decreasing. By contrast, the BMN growth rate is relatively constant in this region. As $\Delta \mu$ increases beyond this region, there is a sudden onset of much larger growth rates, but the resulting crystals are disordered. The growth rate of BMN increases as the temperature is increased (Figs. 517).

We next attempted to model the growth of BMN-BZ $(1-x)\left(\mathrm{Mg}_{1 / 3} \mathrm{Nb}_{2 / 3}\right)+x \mathrm{Zr}$ solid solutions. In the electrostatic Hamiltonian in Eq. (4), tetravalent Zr corresponds to a neutral charge $q_{l}=0$, so sites occupied by $\mathrm{Zr}$ have zero interaction energy. As in the simulations of pure BMN systems, the chemical composition determines the probabilities with which different charge species are adsorbed at the surface. In the initial sub- 


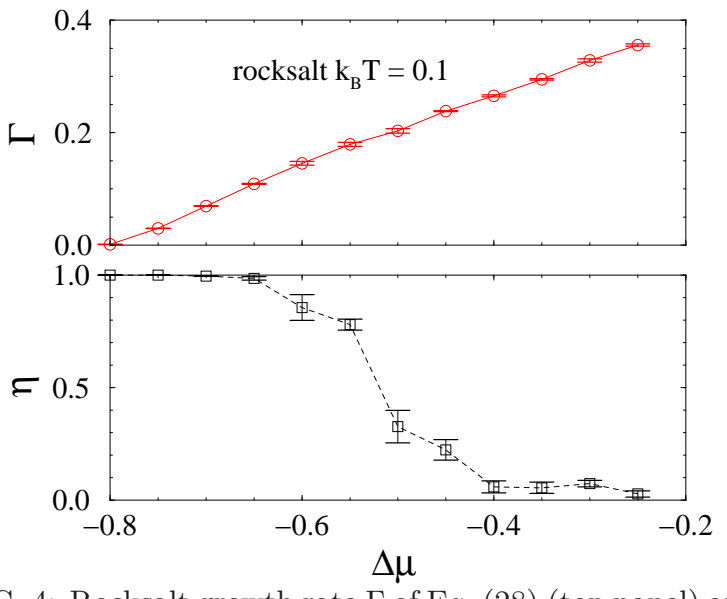

FIG. 4: Rocksalt growth rate $\Gamma$ of Eq. (28) (top panel) and 1:1 order parameter $\eta\left(\boldsymbol{k}=\frac{2 \pi}{a}\left(\frac{1}{2}, \frac{1}{2}, \frac{1}{2}\right)\right)$ (bottom panel) vs. chemical potential. The temperature is $k_{B} T=0.1$ and the growth direction is [001]. A $12 \times 12$ supercell is used, with $1000 \mathrm{MC}$ time steps.

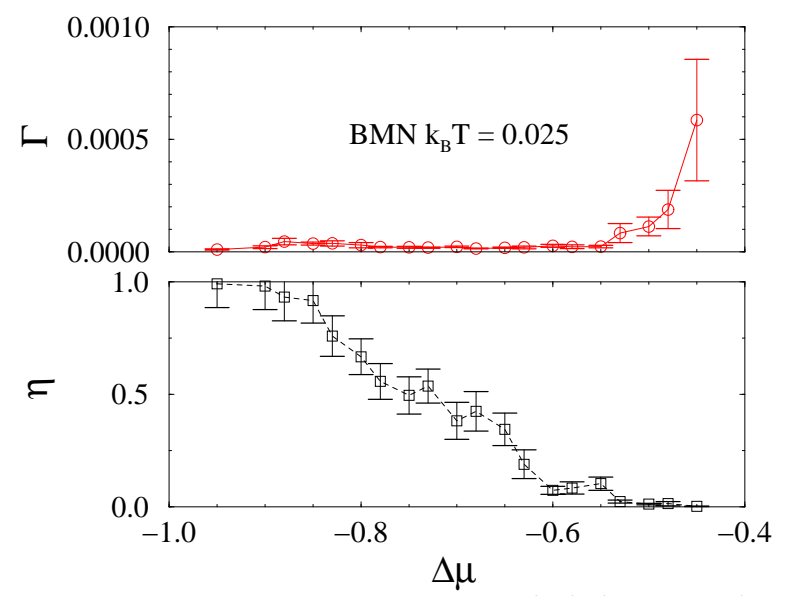

FIG. 5: BMN growth rate $\Gamma$ of Eq. (28) (top panel) and 1:2 order parameter $\eta\left(\boldsymbol{k}=\frac{2 \pi}{a}\left(\frac{1}{3}, \frac{1}{3}, \frac{1}{3}\right)\right)$ (bottom panel) vs. chemical potential. The temperature is $k_{B} T=0.025$ and the growth substrate direction is [1]11]. A $6 \times 6$ supercell is used, with 300,000 MC time steps.

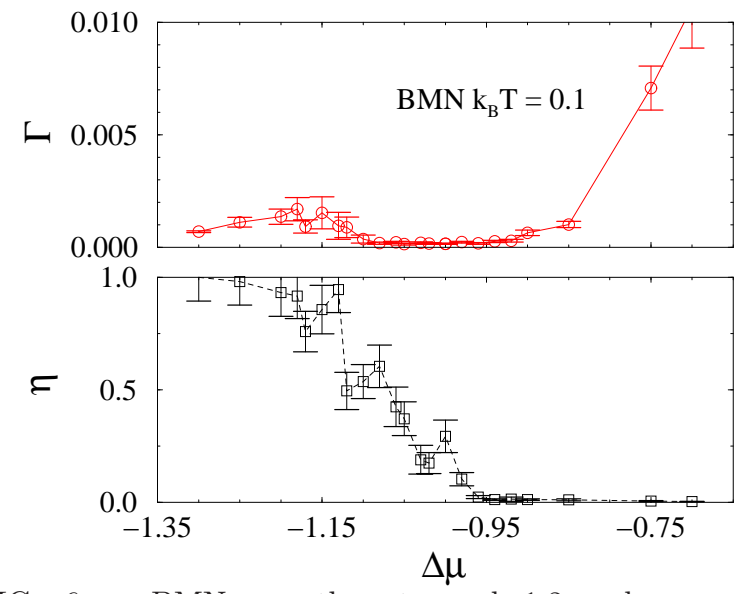

FIG. 6: BMN growth rate and 1:2 order parameter vs.chemical potential. The temperature is $k_{B} T=0.1$. Other parameters are the same as in Fig. 5

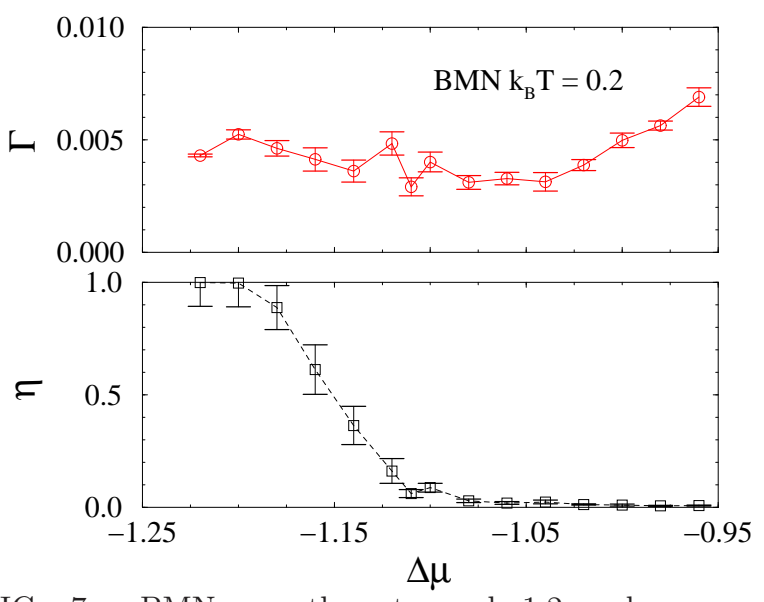

FIG. 7: BMN growth rate and 1:2 order parameter vs.chemical potential. The temperature is $k_{B} T=0.2$. Other parameters are the same as in Fig. 5 .

strate, tetravalent ions with the corresponding concentration were incorporated, using random mixing (next section). With a 1:2-ordered substrate, we studied concentrations $x \sim 10 \%$, with temperatures of $k_{B} T \sim 0.1$ to 0.2 , and varying the chemical potential $\Delta \mu \sim-1.0$ to -0.5 . Very little incorporation of the tetravalent ions occurred. We found similar results with an initially 1:1ordered substrate (random-site model; see below), where a wider range of $x$ was explored. Again the order of the substrate was not sufficient to induce the incorporation of tetravalent ions in the growth phase. Instead the system seemed to favor evaporating the adsorbed tetravalent ions more than the charged particles, to grow pure BMN.

\section{B. Energy Calculations}

To further study the inability to incorporate tetravalent ions at low temperatures, we examined the total energy per particle $\varepsilon_{N}$ of fixed slab configurations of B-site order. A phase separated model, in which all the tetravalent adatoms were situated in the outermost surface layers, was compared with various structural models that incorporated tetravalent ions. In each model, the calculations were performed for two different configurational B-site orderings of the +2 and +5 ions $\left(q_{l}=-2,+1\right.$, respectively). These configurations were the $1: 1$ and $1: 2$ layering along [111] directions, i.e., $[111]_{1: 1}$ and $[111]_{1: 2}$ order, repectively.

The $[111]_{1: 2}$ ordering corresponds to the $x=0$ order of BMN, with a layer of $q_{l}=-2$ alternating with two layers of charge $q_{l}=+1$ along the [111] direction. We chose the $[111]_{1: 1}$ ordering to correspond to the randomsite mode ${ }^{3}$, , which is observed in the BMN-BZ equilibrium simulations for $x>0.05 \stackrel{10,22}{=}$ In the random-site model there are [111] layers of $q_{\boldsymbol{l}}=+1$ alternating with a mixed layer of charges $q_{\boldsymbol{l}}=-2,+1,0$. The random-site model is meant to represent the presence of short-range B-site order from experimental observations. No long- 


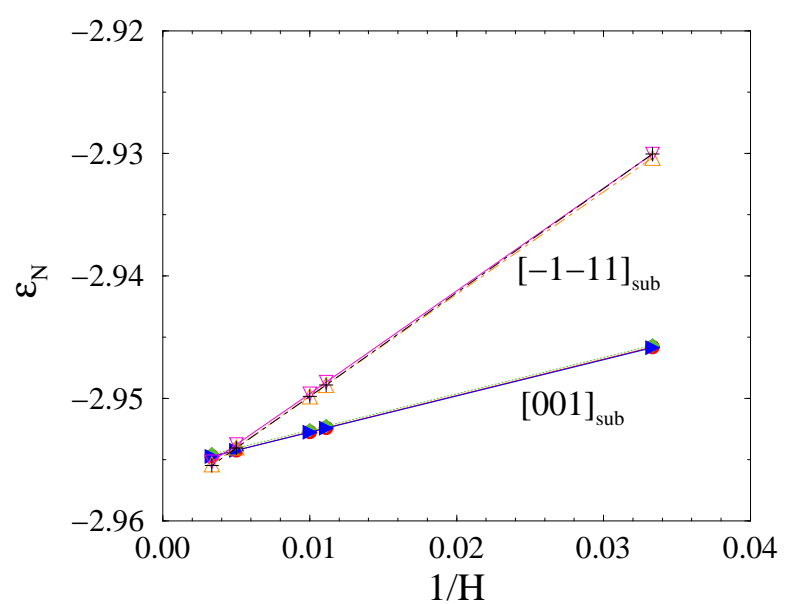

FIG. 8: Total energy per particle for B-site $[111]_{1: 2}$ ordering as a function of slab thickness $1 / H$ and slab crystallographic orientation. Each set has three barely distinguishable curves, corresponding to three lattice sizes: $12 \times 12,15 \times 15$, and $18 \times 18$.

range ordering has been observed. Nevertheless in our simple model here we will fix the ordered $q_{l}=+1$ layers and choose the mixed layers to be a random mixture of $(-2)_{\frac{2}{3}(1-x)}(+1)_{\frac{1}{3}(1-4 x)}(0)_{2 x}$.

We first examine finite-size effects in Fig. 8] which plots $\varepsilon_{N}$ as a function of slab thickness for various 2-D supercells containing no tetravalent ions, for $[111]_{1: 2}$ ordering. Results for [001] and [1111] slabs are shown, both of which correspond to neutral surface layers. As $H \rightarrow \infty$, $\varepsilon_{N} \sim \varepsilon_{N}^{B}+$ const./ $H$ as expected, where the constant $\varepsilon_{N}^{B}$ represents the average bulk value and $H$ is the slab thickness.

Size effects with incorporated tetravalent ions are studied next. Fig. 9plots $\varepsilon_{N}$ for [111] $]_{1: 2}$ ordering as a function of slab thickness for various concentrations of randomly mixed tetravalent ions, using a [1̄111] slab and $15 \times 15$ supercell. These calculations are for a random distribution of +0 (tetravalent) ions replacing -2 or +1 ions in an otherwise perfectly ordered [111 $]_{1: 2}$ slab at each thickness $H$. Within statistical error bars, the asymptotic $H$ dependence is similar to that without tetravalent ions in Fig. 8 Fig. [10 plots $\varepsilon_{N}$ for [111 $]_{1: 1}$ ordering with and without randomly mixed $10 \%$ tetravalent ions. As seen in Fig. $9 \varepsilon_{N}$ rapidly increases with increasing $x$. This is consistent with the inability to incorporate tetravalent ions in the growth simulations on $[111]_{1: 2}$ ordered slabs. By contrast $\varepsilon_{N}$ for $[111]_{1: 1}$ ordering is essentially independent of $x$ within statistical error, as shown in Fig. 10

Fig. 11]plots $\varepsilon_{N}$ as a function of tetravalent concentration $x$ for random-mixing and phase-separation models, showing results for $[111]_{1: 1}$ and $[111]_{1: 2}$ ordered $12 \times 12$ [001] slabs $(H=200)$. For the phase-separation model, the total number of ions includes the outermost layers of tetravalent ions. At $x=0$ the $1: 2$ ordered crystal has a lower energy than the 1:1 ordered crystal, which

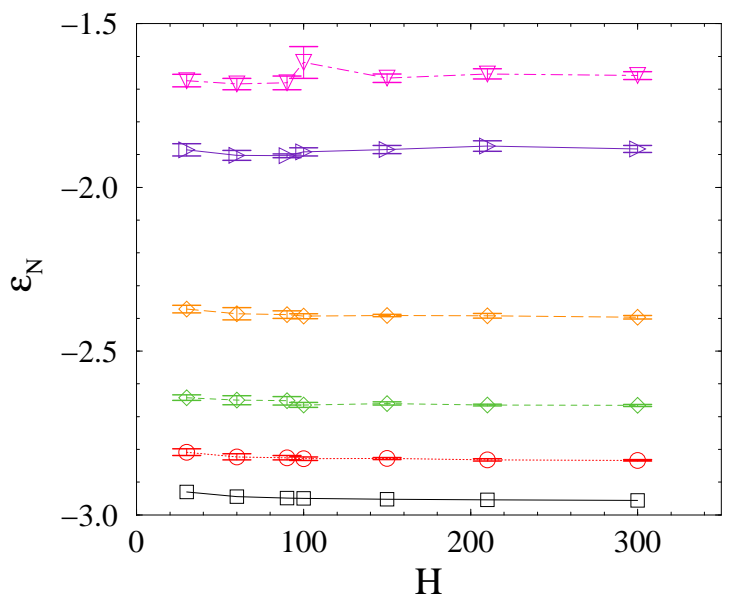

FIG. 9: Total energy per particle for $[111]_{1: 2}$ ordering, as a function of slab thickness $H$ and (randomly mixed) tetravalent concentration $x$. E/ $\mathrm{N}$ increases, as $\mathrm{x}$ increases from $0 \%$ to $2 \%, 5 \%, 10 \%, 15 \%$, and $25 \%$. A [1]11] slab with a $15 \times 15$ supercell was used.

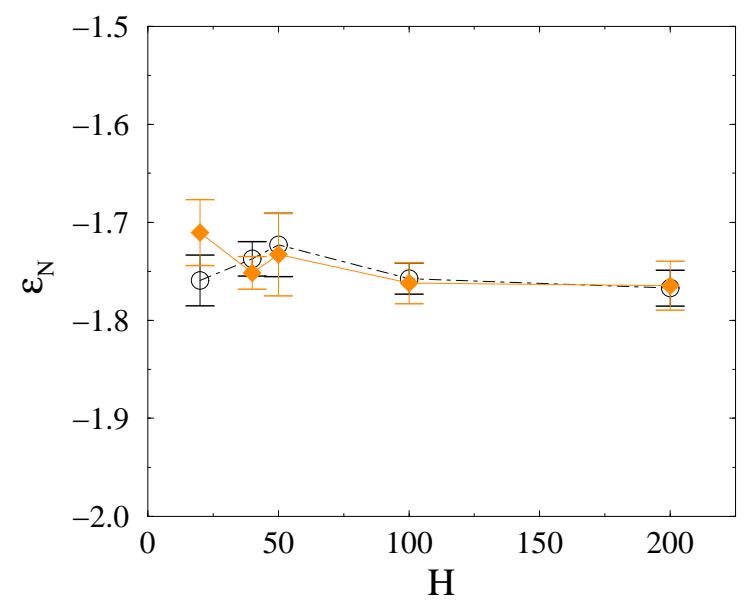

FIG. 10: Total energy per particle vs. slab thickness. Results are shown for $[111]_{1: 1}$ ordering with (dashed line) and without (solid line) 10\% randomly mixed tetravalent ions. A [001] slab with a $12 \times 12$ supercell was used.

is consistent with our results from the growth simulation and with the observed ground state configuration of pure BMN. For random-mixing, $\varepsilon_{N}$ increases linearly with $x$ for $[111]_{1: 2}$ ordering while it is essentially independent of $x$ for $[111]_{1: 1}$ ordering. In the phase-separation model, $\varepsilon_{N}$ increases linearly for both orderings. These results show that phase separation is favored for the $[111]_{1: 2}$ ordering, while random mixing is favored by $[111]_{1: 1}$ ordering.

Fig. [1]illustrates why the growth simulations failed to incorporate tetravalent ions at low temperature. In the electrostatic model, the 1:2 ordered state is the ground state and is optimally ordered. The potential energy between any charge and all other charges in the system is negative. For example, with a $18 \times 18$ slab this poten- 


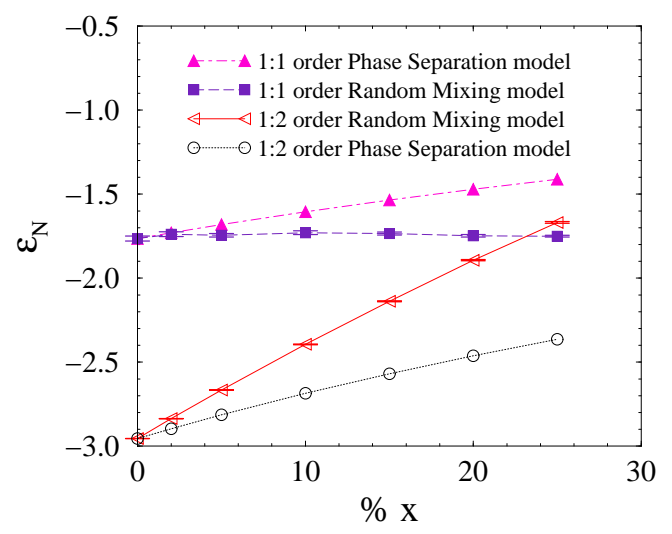

FIG. 11: Total energy per particle vs. tetravalent concentration $x$ for random-mixing and phase-separation models. Results are shown for $[111]_{1: 1}$ and $[111]_{1: 2}$ ordered $12 \times 12[001]$ slabs $(H=200)$.

tial energy is $\sim-5.92$ for a -2 charge and $\sim-1.48$ for a +1 charge. Thus, replacing a charge (either -2 or +1 ) by a neutral tetravalent ion in this state raises the total energy of the system, while a phase-separated configuration in which the tetravalent ion is placed away from the the ordered slab keeps the total energy unchanged. To examine this more closely, we calculated the free-energy $\left(F=\varepsilon_{N}-T S\right)$, where $S$ is the mixing entropy due to the incorporated tetravalent ions. Fig. 12 plots the free-energy as a function of temperature for four concentrations of tetravalent ions. The free energy of the phase-separated 1:2 ordered slabs is constant in our model, because it is perfectly ordered and has vanishing entropy. The free energy of the phase-separated 1:1 ordered slabs decreases with increasing temperature, despite the perfectly ordered outmost layers of tetravalent ions, due to the mixing entropy of the random layers with $-2,+1$, and 0 charges. In all cases in Fig. 12 the phase-separated 1:2 ordered slabs have the lowest free energy at low temperatures, where ordered crystal growth occurs in our simulations, but at temperatures between $k_{B} T \sim 1-2$ the 1:2 ordered and the 1:1 ordered random mixing models start to be favored.

\section{DISCUSSION}

There are striking differences between the growth behavior of the $\mathrm{III}_{1 / 2} \mathrm{~V}_{1 / 2}$ rocksalt ordered structure and the $\mathrm{II}_{1 / 3} \mathrm{~V}_{2 / 3}$ BMN structure. The ordered rocksalt structure forms over a wide range of $\Delta \mu$ (absorption rates) as shown in Fig. 4. By contrast, ordering of the 1:2 structure in BMN type crystals is more difficult to achieve experimentally: ${ }^{23.24}$ When these materials are initially synthesized, they crystallize in a disordered structure. With extended annealing the 1:2 structure is approached..$^{23}$ As discussed by Davies et al. ${ }^{23}$, the initial

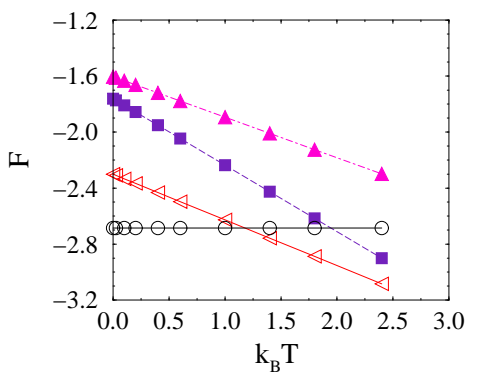

(a)

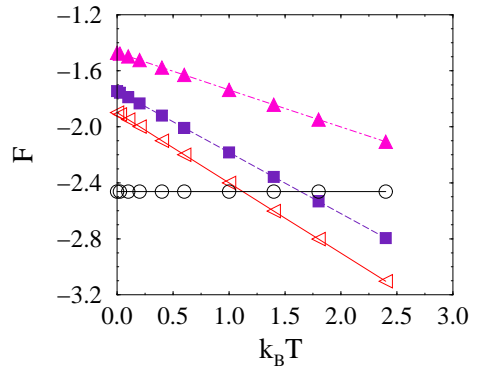

(c)

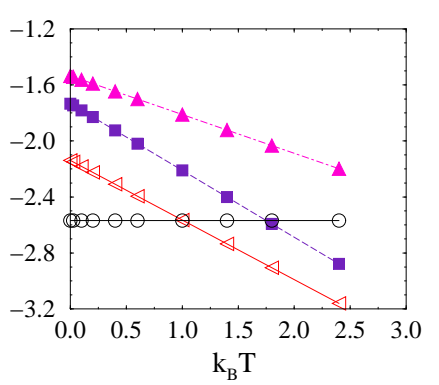

(b)

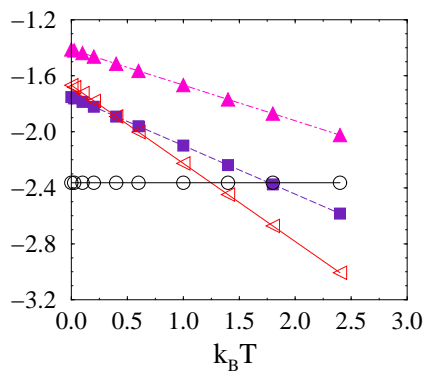

(d)
FIG. 12: Free energy of BMN crystal for (a) $10 \%$ (b) $15 \%$ (c) $20 \%$ (d) $25 \%$ tetravalent concentrations. Symbols have the same meaning as in Fig. [11]

synthesis and processing are controlled by irreversible kinetic processes rather than by thermodynamic factors, and a more correct description of the formation of the $1: 2$ ordered structures is in terms of the nucleation and growth of small ordered domains with increasing annealing time and temperature. Eventually large (>100 nm) 1:2 ordered domains are observed $\stackrel{23,24}{\text { The need for long }}$ annealing times is consistent with our simulations. Figs. 5-7 show that the range of $\Delta \mu$ where ordered 1:2 growth occurs narrows as the temperature increases from $k_{B} T$ $=0.025$ to 0.2 . In this range, the growth rate is approximately constant as a function of $\Delta \mu$. Moreover, when ordered crystal growth occurs, the BMN growth rate is much smaller than that of the rocksalt structure at the same temperature. Highly ordered growth was possible in the BMN simulations but required low temperatures and a delicate balance with the chemical potential. Neither of these requirements is likely to be met under experimental synthesis conditions. At temperatures corresponding to the actual sintering temperature of BMN $\left(k_{B} T \sim 0.5\right)$, large growth rates can be achieved, as shown in Fig. 2, but the growth is highly disordered. The long annealing times allow the slow formation of the 1:2 ordered regions. In our KMC simulations, diffusion processes are excluded so there can be no annealing. We also note that the growth rate was sensitive to the slab orientation. For example, we found that growth rate along [1]11] direction was almost an order of magnitude larger than that along [001].

Our results are also qualitatively consistent with the 
long experimental history of failed attempts to coarsen the 1:1 ordered nanoscale domains in PMN type crystals. Prior to the experiments of Akbas and Davies ${ }^{24}$, the 1:1 ordered regions were apparently limited to nanoscale size and represented only a small volume fraction of the crystal. The space-charge model, which was invoked to explain this behavior, hypothesized that the 1:1 ordered regions arose from a rocksalt ordering of the -2 and +1 B-site charges, implying charge-imbalanced 1:1 domains. The apparently limited size of these domains could be explained by the rapidly increasing energy of larger domains due to Coulomb repulsion. With careful annealling at much higher temperatures than had previously been tried, however, some fully 1:1 ordered crystals were synthesized 24 . Our calculations show that long-range ionic interactions favor the growth of disordered crystals, and ordering occurs only after annealing. Moreover, ionic interactions appear to favor the 1:2 ordering. However, entropic contributions to the free energy and short-range covalent interactions tend to favor 1:1 ordering. Covalent bonding is negligible for $\mathrm{Ba}$ ions but very important for $\mathrm{Pb}$ ions. Thus there is a delicate competition between 1:2 and 1:1 ordering for doping with small concentrations of the tetravalent ions in (1-x)BMN$\mathrm{xBZ}$ and (1-x)PMN-xPT. In (1-x)BMN-xBZ, there is a crossover from 1:2 to $1: 1$ ordering as $x$ increases to about $5 \%$. While in (1-x)PMN-xPT, the stronger short-range covalent bonding of $\mathrm{Pb}$ favors 1:1 ordering at all concentrations.

For pure systems, our minimal paradigm for growth simulations captures the differences in growth rate and ordering between rocksalt-type and BMN-type crystal growth. This indicates that the simple ionic model is a reasonable starting point for describing the growth of perovskite solid solutions. More direct and quantitative comparisons with experiment will require additional ingredients such as short-range interactions and the inclusion of diffusive processes.

For systems with tetravalent ions, our results show that the ground state is a phase-separated state of tetravalent ions and 1:2 ordered BMN over a wide range of tetravalent compositions. On the other hand, equilibrium simulations of the ionic model ${ }^{10.22}$ suggest that for $x>0.05$ the $1: 1$ ordering is preferred, with no phase separation. Several factors distinguish these calculations, which likely have to do with the apparent contradiction in their observations. The first is the difference in the nature of the simulations. In our growth simulation, tetravalent ions are allowed to evaporate from the crystal, which facilitates phase separation. The equilibrium calculations were done in the canonical ensemble with the tetravalent ions mixed in, where it is more difficult to detect phase separation without large simulation cell sizes. Our simulations were at lower temperatures where ordered growth could be induced by tuning the chemical potential $\Delta \mu$ (absorption rate). At these temperatures the system is essentially in the ground state, as Fig. 3 shows. Incorporation of tetravalent ions could be induced at larger $\Delta \mu$, which is expected as adsorption dominates evaporation, but in this case random growth occurs. Secondly, since our $[111]_{1: 1}$ structure is an artificial model of random mixing of $-2,+1$, and neutral charges in one layer and perfectly ordered +1 in another, its energy must be higher than the actual 1:1 structure achieved in the equilibrium simulations. This means that the actual cross-over of the random-mixing $[111]_{1: 1}$ structure will occur at lower temperatures. Indeed, the $k_{B} T \sim 0.25$ equilibrium calculations show $[111]_{1: 1}$ ordering for concentrations $x$ greater than about 0.05 . Thus the absence of phase separation in the equilibrium calculations might be due to a lower free-energy than our estimate in Fig. 12 from the artificial random-site structure. Our results combined with the equilibrium calculations therefore suggest the following picture of the equilibrium state of the ionic model. In the ground state phase-separation takes place for $x>0$. Beyond some $x$-dependent critical temperature tetravalent ions are incorporated, most likely in a structure that favors 1:1 order.

To determine if the new phase (phase-separation) at low temperatures that we have found is realistic for these alloys, the ionic model must be improved. One possibility is first-principles based $H_{\text {eff }}$, which have shown great promise in describing ferroelectrics and simple solidsolutions ${ }^{25}$. Like the Ising model these $H_{\text {eff }}$ project out what are considered to be the most important ionic degrees of freedom. In addition to the long-range Coulomb interaction, short-range interactions are also included. The $H_{\text {eff }}$ parameters are fitted to the results of a set of first-principles density-functional calculations, so there is effectively no experimental input (except sometimes the average crystal volume). The simplified form of $H_{\text {eff }}$ for ferroelectrics and ferroelectric alloys has permitted simulations of equilibrium properties on thousands of atoms as a function of temperature and applied external electric field. A main difficulty in applying these in a growth simulation is computational cost, which has typically required fixed distributions of B-site ions even in equilibrium simulations of solid-solutions. In our kinetic Monte Carlo model, another possibly important factor that is not included is surface diffusion. Coupled with the solidon-solid restriction, the simulation is severely limited in its ability to "heal" disorder, and these approximations may have contributed to low ordered growth rates and raised the critical temperature for phase separation. Removal of these restrictions would improve the model and increase its applicability.

\section{SUMMARY}

The growth of the technologically important BMN type perovskite alloys was studied by kinetic Monte Carlo using an ionic model. An enhanced KMC algorithm was formulated to treat long-range Coulomb interactions efficiently. We found that this minimal paradigm was capable of describing ordering features of the growth of pure 
BMN and PMN type single crystals. The largest growth rates were observed along the [11ㅣ] direction, but best ordered growth rates are substantially less than those of rocksalt. Highly ordered growth was possible, but required very low temperatures and a delicate balance with the chemical potential. For mixed systems such as BMNBZ, we found that the $T=0$ ground state of the model was one in which tetravalent ions phase separate from a 1:2 ordered pure system. As a result, little incorporation of tetravalent ions occurs in the growth process at low temperatures. At higher temperatures, tetravalent ions can be incorporated, but the resulting crystals show no chemical ordering. The tendency of the purely ionic model to favor phase separate was further studied using free energy calculations determined from $\mathrm{T}=$ 0 total energy calculations and including a mixing entropy. This indicated that, if diffusive mechanisms were included, chemical orderings consistent with those found in equilibrium studies could develop at the higher temperatures characteristic of realistic alloy synthesis.

\section{Acknowledgments}

Support from ONR (N000149710049 and N000140110365), NSF (DMR-9734041), and Research Corporation are gratefully acknowledged. We would like to thank C. Tahan, T.J. Walls, and P. Larsen for enjoyable and productive collaborations and for their contributions in early stages of this work.

\section{APPENDIX A: COULOMB POTENTIAL}

The 2-D Ewald potential, is given as the sum of three terms

$$
v\left(\boldsymbol{l}^{\prime}-\boldsymbol{l}\right)=v_{1}\left(\boldsymbol{l}^{\prime}-\boldsymbol{l}\right)+v_{2}\left(\boldsymbol{l}^{\prime}-\boldsymbol{l}\right)+v_{s}\left(\boldsymbol{l}^{\prime}\right)
$$

where $v_{1}$ and $v_{2}$ are due to $\rho_{1}(\boldsymbol{r})$ and $\rho_{2}(\boldsymbol{r})$, respectively in Eq. (13), and $v_{s}$ is the correction for the interaction of the point charge $q_{l^{\prime}}$ with its own Gaussian density $q_{\boldsymbol{l}^{\prime}} g\left(\boldsymbol{r}-\tilde{\boldsymbol{l}}^{\prime}\right)$ in $\rho_{2}(\boldsymbol{r})$.
To calculate $v_{1}\left(\boldsymbol{l}^{\prime}-\boldsymbol{l}\right)$ we place, for consistency, the $(\boldsymbol{R} \neq 0) q_{\boldsymbol{l}^{\prime}}$ images at their vertical projections onto the plane of the $q_{\boldsymbol{l}}$ sublattice. $v_{1}\left(\boldsymbol{l}^{\prime}-\boldsymbol{l}\right)$ is then given by

$$
\begin{aligned}
v_{1}\left(\boldsymbol{l}^{\prime}-\boldsymbol{l}\right)= & q_{\boldsymbol{l}} \sum_{\boldsymbol{R}} \frac{\operatorname{erfc}\left(\sqrt{\alpha}\left|\boldsymbol{l}^{\prime}-\boldsymbol{l}-\boldsymbol{R}\right|\right)}{\left|\boldsymbol{l}^{\prime}-\boldsymbol{l}-\boldsymbol{R}\right|} \\
& -q_{\boldsymbol{l}} \sum_{\boldsymbol{R} \neq 0} \frac{\operatorname{erfc}\left(\sqrt{\alpha}\left|\boldsymbol{l}^{\prime}-\tilde{\boldsymbol{l}}^{\prime}-\boldsymbol{R}\right|\right)}{\left|\boldsymbol{l}^{\prime}-\tilde{\boldsymbol{l}}^{\prime}-\boldsymbol{R}\right|} .
\end{aligned}
$$

The mathematical form of this contribution is identical to its 3-D counterpart, except that the sum is over $2-\mathrm{D}$ rather than 3-D direct-lattice vectors $\boldsymbol{R}$.

The 2-D planewave expansion of $\tilde{\rho}_{2}^{\left(\boldsymbol{l}, \boldsymbol{l}^{\prime}\right)}(\boldsymbol{r})$ in Eq. (17) is given by

$$
\begin{array}{r}
\tilde{\rho}_{2}^{\left(\boldsymbol{l}, \boldsymbol{l}^{\prime}\right)}(\boldsymbol{r})=q_{\boldsymbol{l}}\left(\frac{\alpha}{\pi A^{2}}\right)^{1 / 2} e^{-\alpha\left(z-l_{z}\right)^{2}} \\
\sum_{\boldsymbol{G} \neq 0} e^{-G^{2} / 4 \alpha}\left[e^{-i \boldsymbol{G} \cdot \boldsymbol{l}_{\alpha p}}-e^{-i \boldsymbol{G} \cdot \boldsymbol{l}_{\alpha p}^{\prime}}\right] e^{i \boldsymbol{G} \cdot \boldsymbol{r}_{p}}
\end{array}
$$

where we have used the fact that $\tilde{\boldsymbol{l}}_{z}^{\prime}=\boldsymbol{l}_{z}$. Substituting into Eq. (8) and using Eq. (11), yields:

$$
v_{2}\left(\boldsymbol{l}^{\prime}-\boldsymbol{l}\right)=\sum_{\boldsymbol{G} \neq 0} \frac{\pi}{A G}[f(G)-f(-G)]\left[e^{i \boldsymbol{G} \cdot\left(\boldsymbol{l}_{p}^{\prime}-\boldsymbol{l}_{p}\right)}-1\right],
$$

where

$$
f(x) \equiv e^{x\left(l_{z}^{\prime}-l_{z}\right)} \operatorname{erfc}\left(\frac{2 \alpha\left|l_{z}^{\prime}-l_{z}\right|+x}{2 \sqrt{\alpha}}\right) .
$$

Finally, the correction for the interaction of the point charge $q_{l^{\prime}}$ with its own Gaussian density is given by:

$$
v_{s}\left(\boldsymbol{l}^{\prime}\right)=\frac{\operatorname{erf}\left(\sqrt{\alpha}\left|\boldsymbol{l}^{\prime}-\tilde{\boldsymbol{l}}^{\prime}\right|\right)}{\left|\boldsymbol{l}^{\prime}-\tilde{\boldsymbol{l}^{\prime}}\right|}
$$

As verified by direct calculation, the sum of these three terms in independent of the parameter $\alpha$. For efficiency, $v\left(\boldsymbol{l}^{\prime}-\boldsymbol{l}\right)$ is stored as a look-up table.
${ }^{1}$ K. Uchino, in Piezoelectric Actuators and ultrasonic Motors (Kluwer Academic Publishers, Boston, 1996).

2 S.-E. Park and T. E. Shrout, J. Appl. Phys. 82, 1804 (1997).

3 M. A. Akbas and P. K. Davies, in Solid State Chemistry of Inorganic Materials, edited by P. Davies, A. J. Jacobson, C. Torardi, and T. A. Vanderah (Materials Research Society, 1997), vol. 453, p. 483.

${ }^{4}$ L. Chai, M. Akbas, P. Davies, and J. Parise, Materials Research Bulletin 32, 11 (1997).

5 M. A. Akbas and P. K. Davies, J. Am. Ceram. Soc. 80, 2933 (1997).
${ }^{6}$ M. A. Akbas and P. K. Davies, Journal of Materials Research 12, 2617 (1997).

7 E. Husson, Mat. Res. Bull. 23, 357 (1988), ibid., 25, 539 (1990).

8 J. Chen, J. Am. Cer. Soc. 72, 593 (1989).

9 A. B. Bortz, M. H. Kalos, and J. L. Lebowitz, J. Comput. Phys. 17, 10 (1975).

10 L. Bellaiche and D. Vanderbilt, Phys. Rev. Lett. 81, 1318 (1998).

11 K. A. Jackson, G. H. Gilmer, and D. E. Temkin, Phys. Rev. Lett. 75, 2530 (1995).

12 V. A. Shneidman, K. A. Jackson, and K. M. Beatty, J. 
Chem. Phys. 111, 6932 (1999).

13 See, for example, K. Binder and M. H. Kalos, in Monte Carlo Methods in Statistical Physics, edited by K. Binder (Springer-Verlag, Berlin, Berlin, 1986), 2nd ed.

14 C. Tahan, M. Suewattana, P. Larsen, Shiwei Zhang, and H. Krakauer, in Fundamental Physics of Ferroelectrics 2001, edited by H.Krakauer (AIP conf. Proceedings, 2001), vol. 582 , p. 118.

15 S. W. de Leeuw, J. W. Perram, and E. R. Smith, Proc. R. Soc. Lond. A373, 27 (1980).

16 S. W. de Leeuw and J. W. Perram, Molec. Phys. 37, 1313 (1979).

17 B. Nijober and F. de Wette, Physica 23, 309 (1957).

18 D. M. Ceperley, Phys. Rev. B 18, 3126 (1978).
19 E. R. Smith, Physica 120A, 327 (1983).

20 A. Levi and M. Kotrla, J. Phys.: Cond. Matt. 9, 299 (1997).

21 See, for example, M. H. Kalos and P. A. Whitlack, Monte Carlo Methods (John Wiley and Sons, New York, 1986).

22 Z. Wu and H. Krakauer, Phys. Rev. B 63, 137113 (2001).

${ }^{23}$ P. Davies, J. Tong, and T. Negas, J. Am. Ceram. Soc. 80, 1727 (1997).

24 M. A. Akbas and P. K. Davies, J. Am. Ceram. Soc. 81, 670 (1998).

25 See, for example, R. E. Cohen, ed., Fundamental Physics of Ferroelectrics 2002, vol. 535 (AIP conf. Proceedings, 2002). 